\title{
The Steady State Challenge for Soft X-Ray Diagnostics on Wendelstein 7-X Stellarator
}

\author{
H. Thomsen ${ }^{\mathrm{a}}$, T. Broszat ${ }^{\mathrm{a}}$, P. Carvalho ${ }^{\mathrm{b}}, \mathrm{S} . \mathrm{Mohr}^{\mathrm{a}}$, A. Weller ${ }^{\mathrm{a}}$ and M.Y. Ye \\ ${ }^{a}$ Max-Planck-Institute für Plasmaphysik, Wendelsteinstr. 1, 17491 Greifswald, Germany \\ ${ }^{b}$ Associação Euratom/IST Centro de Fusão Nuclear, Instituto Superior Técnico 1049-001 Lisboa, Portugal
}

\begin{abstract}
The steady state operation of Wendelstein 7-X stellarator presently under construction in Greifswald poses special challenges to the diagnostics development $[1,2]$. A critical issue is the heat load on plasma facing components $\left(\sim 500 \mathrm{~kW} / \mathrm{m}^{2}\right)$ over a long discharge time (up to $30 \mathrm{~min}$ ), which leads to the necessity of active cooling. As result, the design of the 400 channel soft X-Ray Multi Camera Tomography System (XMCTS) [2,3] has to cope with dark currents and amplifier drifts due to the heating of active components like photo diodes and in-vessel preamplifiers. In order to allow for a quantitative measurement of dynamic drifts and offsets, a shutter system and blind diodes are considered to compensate these effects. Another important issue is the large amount of data gathered by the XMCT system during long pulse discharges. A fast but less precise online reconstruction is planned, which will give information on the plasma shape and position on a human time scale. The two options under investigation are a Cormack-Inversion method and an approach based on neural networks [4]. Dependent on the available hardware, as much information as possible should be stored for more accurate offline-analysis. An intelligent way of marking interesting data is required. In case that the steady-state storage of all measured data is not feasible, at least this marked data will be stored in high time resolution.
\end{abstract}

Keywords: steady state diagnostics, Soft X-Ray tomography.

PACS: $52.55 . \mathrm{He}$, 52.70.La, 07.05.Fb

\section{INTRODUCTION}

The challenges posed by long pulse discharges to a plasma diagnostic [1,2] can be divided into three topical groups, which are: protection \& safety, measurement quality and steady state data acquisition \& online analysis. In Fig. 1 several constraints are mentioned. Since the soft X-Ray Multi Camera Tomography System (XMCTS) [3] will be located inside the plasma vessel of Wendelstein 7-X stellarator, a heat protection is required to withstand power loads of up to $500 \mathrm{~kW} / \mathrm{m}^{2}$. The system consists of 20 pinhole cameras, located in a single poloidal plane (a triangular shaped plasma plane without divertor installations restricting plasma observation). In Fig. 2 a schema of the XMCTS' major components is shown.

The cameras are equipped with photo diode arrays (18 active +4 blind diodes) which are connected to nearby preamplifiers via custom-made multi-pin vacuum feed throughs. The electrical bandwidth of the system will be $500 \mathrm{kHz}$. Beryllium filters are used to block low energetic photons $(<1 \mathrm{keV})$. The accurate knowledge of the camera sight lines is essential for an accurate tomographic reconstruction of the signals. The XMCTS system will provide information about the shape and position of the plasma as well as location and frequency of MHD modes. Since this information might be used within online control schemes, methods of real-time inversion are presently under investigation [4]. 


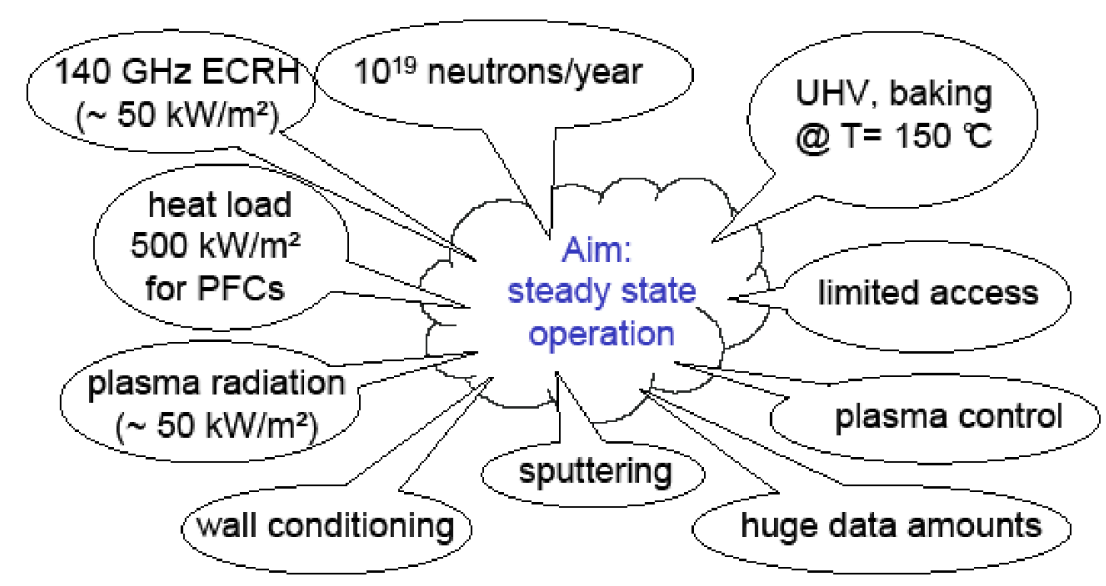

FIGURE 1. Installing a steady state diagnostics on Wendelstein $7-\mathrm{X}$ requires the consideration of various constraints (PFC: plasma facing components).

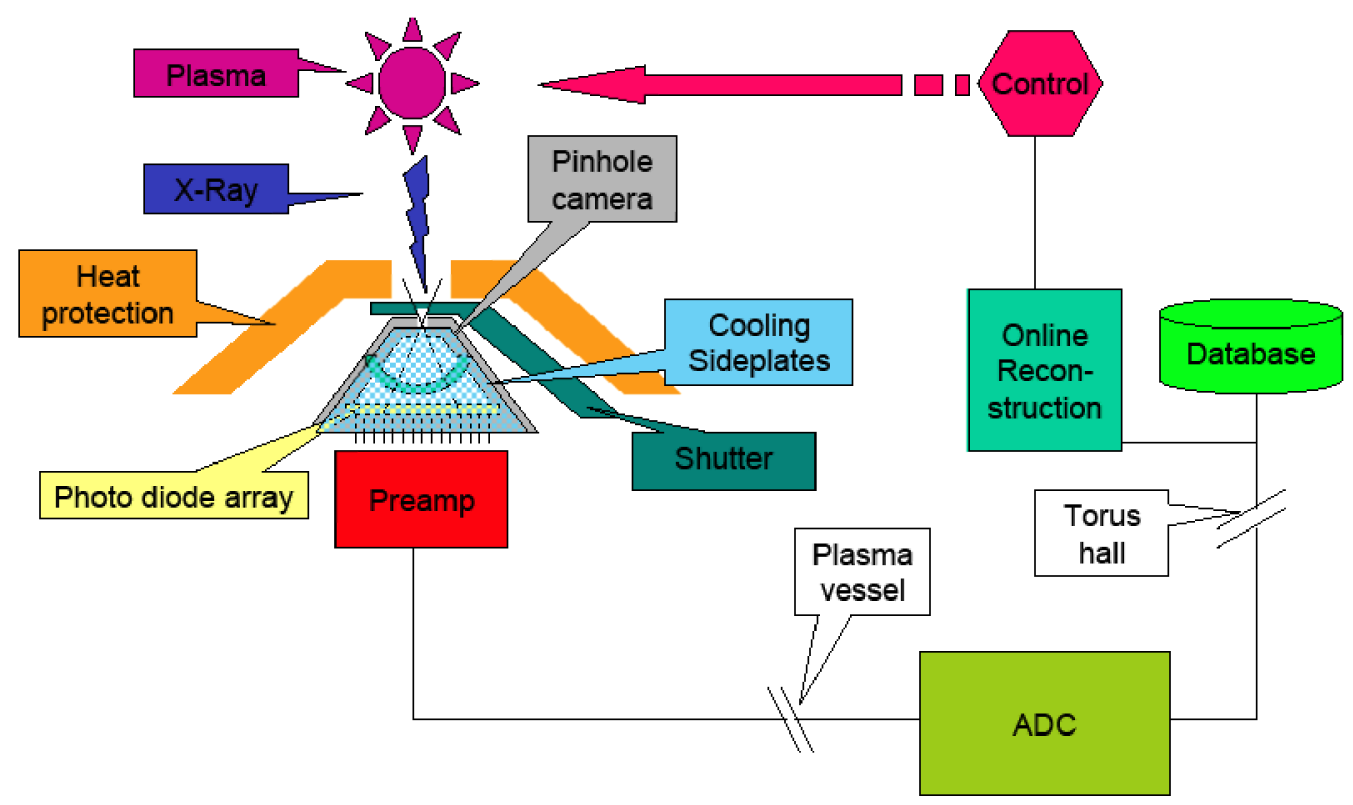

FIGURE 2. Schematic drawing of the major components of the XMCTS diagnostics.

\section{HEAT PROTECTION}

The design of the heat protection is finished and finite element modelling (FEM) calculations were performed to investigate the validity of the concept with respect to material constraints. As an example, the utilized $\mathrm{CuCrZr}$-cooling structure will be damaged above temperatures of $450^{\circ} \mathrm{C}$.

Since the areas facing the XMCTS-diagnostics heat up to a temperature of $850{ }^{\circ} \mathrm{C}$, the cameras must be thermally insulated from these hot areas and additional water cooling is necessary to remove radiation heat. In order to keep the dark current of the utilised photo diodes sufficiently low, the maximum allowable temperature of the diode array is $30^{\circ} \mathrm{C}$. According to FEM calculations (cf. Fig. 3 and Ref. [5]) and preliminary laboratory experiments, this can be achieved with water cooled $\mathrm{CuCrZr}$ side plates and a water cooled base plate around the camera housing.

An important issue for all in vessel components is a very good reliability, since service intervals breaking the machine vacuum will be rare (once a year). However, it is expected that components of the XMCTS must be exchanged in regular intervals. For instance, the diode array and the preamplifier electronics are exposed to a neutron rate of up to $10^{19}$ neutrons per year, which will degrade measurement quality and even cause failure of the photo diodes. Therefore, the heat protection allows to exchanging critical components. 

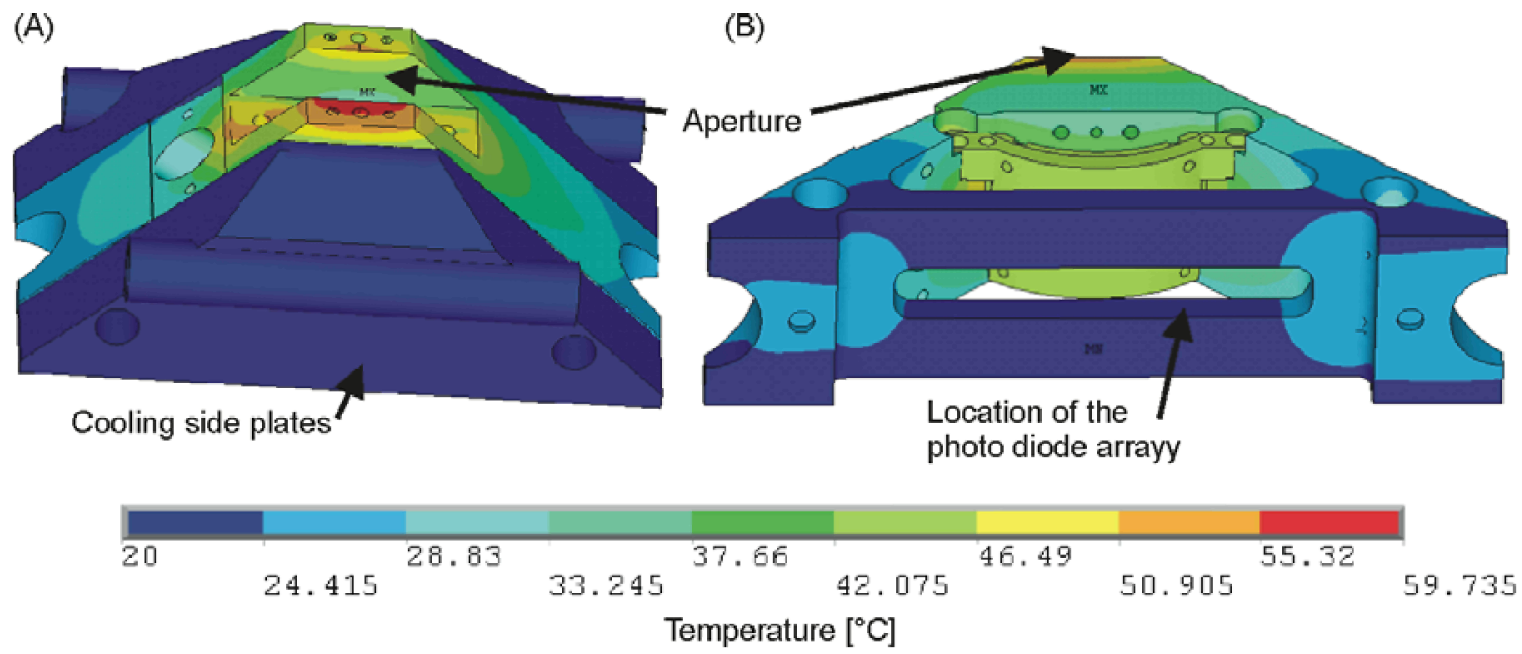

FIGURE 3. Results from finite element calculations [5]. (a) Side view on a XMCTS camera with the cooling side plates (dark blue, CuCRZr with brazed stainless steel water pipes). (b) View on the pinhole camera (without side plates) from below; in front is the part where the photo diode array is located. The heating is by radiation from the heat protection (not shown, reaches up to $850^{\circ} \mathrm{C}$ with a heat load of $500 \mathrm{~kW} / \mathrm{m}^{2}$ on the heat protection) and direct plasma radiation through the camera aperture.

\section{OFFSET COMPENSATION MEASURES}

The cooling of the camera and possibly additional peltier cooling elements beneath the photo diode array ensure a low offset due to dark current and drifts of the operational amplifier circuits. However, since the reconstruction of radiation distribution profiles in the poloidal plane of the diagnostics requires accurate DC signal values, two additional measures of offset compensation are foreseen. First, four of the (outermost) diodes on the photo array have a shadowing lid (Fig. 4a), so that they don't collect any soft X-ray radiation from the plasma. The dark currents and their temperature drifts can thus be detected by these diodes and be corrected in the active channels, presuming the same drift in the active diodes. Second, a fast shutter system should be installed which allows to block the soft X-ray radiation from the plasma (Fig. 4b). The active channels' offsets can be detected while the shutter is closed. The offset profile could either be digitally stored and subtracted or compensated by an analogue sample-and-hold circuit in the main amplifiers.

During a long Wendelstein 7-X discharge of up to 1800 seconds, several shutter closures might be performed in order to monitor the offset behaviour and compensate accordingly. Closing the shutter will also be necessary during wall conditioning phases (boronisation) in order to avoid sputtering on the filters.

(A)

(B)

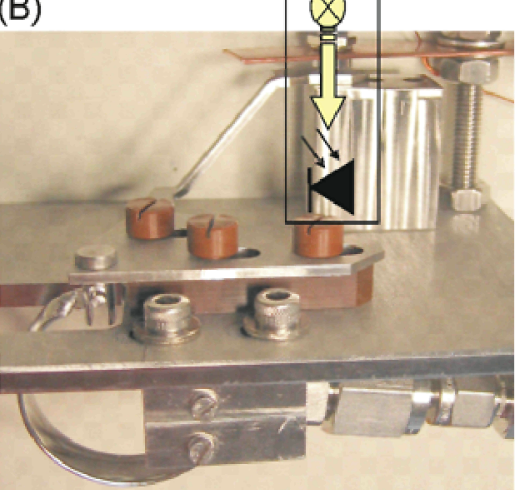

FIGURE 4. Hardware for offset compensation: (A) photo diode array with 4 blind diodes and (B) test setup for the shutter. The shutter consists of a manometer spring tube and a moving lever. For the tests, a photoelectric relay was used (sketched on top of the photograph).

\section{RESULTS FROM SHUTTER TESTING}

The present shutter design is under testing in the laboratory. The design is based on an industrial manometer spring, which moves a lever over the camera aperture to shadow incoming radiation, cf. Fig 4. Pressurised air is used for the shutter operation. The test bed is installed inside a vacuum chamber. Duty cycles are performed at the frequency of $1 \mathrm{~Hz}$. The proper closure of the shutter is monitored by a photoelectric relay. The test system is interlocked by the pressure gauge of the vacuum chamber. A leakage of the shutter inside the vacuum chamber 
immediately aborts the test cycles and stops the counter. A manometer spring without the lever lasted for more than 1 million cycles. With the realistic setup and an increased pressure difference of 5 bar, 250000 duty cycles are a typical number. The leakage appears without any sign of warning in advance. It is therefore not possible to formulate a trigger condition to mark an expected failure time, but the high number of duty cycles seems to be sufficient for an operation time of more than ten years. The tests will be refined in the future to take into account effects of heating of the components. The vacuum chamber contains a ceramic heater for these purposes.

\section{DATA ANALYSIS}

Another important issue is the large amount of data gathered by the XMCT system during long pulse discharges. For a Wendelstein 7-X discharge of $1800 \mathrm{~s}, 1.4 \mathrm{~TB}$ of data are collected for the 400 channels with an envisaged sampling rate of 1 MSamples/s. The strategy to cope with these high data rates is twofold: a (less precise) online reconstruction is planned with a reduced set of channels and with an output rate at human time scale of several reconstructions per second and an offline reconstruction based on constrained regularization methods.

It should be noted here, that it is not yet clear, whether the streaming of the above mentioned data rates to the database is feasible. If that is not the case, the results from the online reconstruction could be used (possibly in conjunction with a novelty detection analysis) to identify interesting time ranges and trigger the transfer of the complete data set within these ranges. In this case, the data acquisition must allow post-triggering. The online reconstructions contain information on the plasma shape and position which can be used for machine operation control (cf. Fig. 2). The two options for online reconstruction under investigation are a Fourier-Bessel inversion method and an approach based on neural networks [4]. The neural networks seem to be faster and more reliable for the Wendelstein 7-X geometry (the Fourier Bessel method is more appropriate for circular plasma crosssections).

\section{SUMMARY}

Steady state discharge operation in the Wendelstein $7-\mathrm{X}$ stellarator poses various challenges to the diagnostics design of the XMCTS. A major constraint is the reliability of in-vessel components due to rare access and service intervals. The design foresees an easy exchange of critical components (e.g., due to neutron bombardment). The diagnostic must be protected from the heat fluxes from the targets and a sufficient cooling setup is required in order to achieve small offset. An active compensation with passive diodes is planned. Another reference for compensation is possible using a shutter for shadowing active diodes from the soft X-ray radiation. The shutters serve also a second purpose, which is the protection of the filters during wall conditioning. Furthermore, a light source for in-situ calibration will be installed in each camera.

The huge data amounts and data rates collected during long pulse discharges are an important field for further investigations. The initial study on online reconstruction methods shows promising results. Especially the neural network inversion has a high potential, since one could train it specifically to plasma physics parameters of interest, e.g., plasma-beta. Neural networks are also good candidates for novelty detection purposes, as will be required for the automated identification of interesting time intervals within the collected data.

\section{REFERENCES}

1. H-J Hartfuss, R König and A Werner, Diagnostics for steady state plasmas, Plasma Phys. Control. Fusion 48 (2006) R83R150

2. R. König et al., this conference.

3. A Weller, SMohr and C Junghans, Concepts of X-ray diagnostics for Wendelstein 7-X, Rev. Sci. Instrum., 75 (2004) 3962-3965

4. P. Carvalho, H. Thomsen et al., this conference.

5. M.Y. Ye, A. Werner et al., this conference. 